CRÍTICA, Revista Hispanoamericana de Filosofía

Vol. XXVIII, No. 83 (agosto 1996): 41-73

\title{
IDENTIDAD, CONSTITUCIÓN Y SUPERVENIENCIA
}

Montserrat Bordes Solanas

Grupo de Epistemología y Análisis

Universidad de Barcelona

En este artículo es mi intención presentar el núcleo de la disputa en torno a las nociones de identidad y constitución tal y como se ha retomado a partir de la controversia entre M. Johnston (1992) y H. Noonan (1993). En primer lugar expondré las dos posibles respuestas básicas que se ofrecen ante los problemas que se plantean y los presupuestos con respecto al carácter modal y temporal de la relación de identidad que suelen acompañarlas. Seguidamente argumentaré que una de esas respuestas se compromete con cierto principio de coincidencia y que viola un principio de superveniencia cuya validez no cabe (incluso más allá de prima facie) poner en duda. Finalmente señalaré las ventajas que, desde la teoría rival, se ofrecen a la hora de abordar estas cuestiones.

\section{I}

Toda teoría ontológica debe reconocer que hay una estrecha relación entre la identidad y la constitución material de un objeto. ${ }^{l}$ Qué sea un objeto depende en parte de qué materia lo constituya. Dicho en otras palabras, la materia es

${ }^{1}$ Una buena introducción técnica a la lógica de la constitución puede hallarse en P. Simons (1987, pp. 210-247). 
causa aristotélica, restringe el conjunto de poderes causales del objeto que determinan su pertenencia a un sortal. (La clásica forma aristotélica.)

Desde luego, tener la misma materia no constituye un criterio de identidad. Los isómeros tienen, a pesar de ser diferentes, la misma materia específica; en diferentes momentos de tiempo, la misma materia numérica puede conformar distintos objetos. Qué sea un objeto depende en parte de su forma o distribución material.

Ésta es la parte no controvertida de la historia. La parte controvertida podría ilustrarse así. Llamemos 'María' a cierta estatua femenina de arcilla que estamos observando y 'Marta' al pedazo de arcilla con que está hecha. 'María' y 'Marta' son nombres, pues, de objetos persistentes. ${ }^{2}$ Existen tres tipos de casos básicos que es imprescindible distinguir con el fin de exponer claramente las posibles respuestas al problema en cuestión. En términos del ejemplo citado serían éstos:

$\alpha$. María es elaborada como resultado de la unión de dos piezas separadas de arcilla. La pieza resultante de la unión es Marta. El escultor, decepcionado ante su obra, intacta hasta entonces, destruye parcialmente la estatua al quebrarle el brazo izquierdo, que se desprende de ella al instante siguiente.

$\beta$. Como en $\alpha$, sólo que el escultor, más exigente y colérico, destruye la estatua completamente haciéndola añicos.

$\gamma$. Se elabora María como en el caso $\beta$, poniendo el nombre de 'Marta' a la pieza de arcilla, en la medida en que mantiene las características de estatua de María.

${ }^{2}$ Un objeto $O$ persiste de $t$ a $t^{\prime}$ si y sólo si $O$ existe en $t$ y en $t^{\prime}$. 
En el caso $\alpha$, la estatua, a partir del momento de su elaboración, está constituida por la pieza de arcilla: Marta y María tienen entonces exactamente los mismos límites espaciales y son cualitativamente idénticas, lo cual parece legitimarnos a afirmar que son entonces numéricamente idénticas. Sin embargo, lo que hace que esta supuesta identidad sea discutible es el hecho de que ambas tienen diferentes propiedades temporales: María pierde un brazo, pero no Marta, ya que la pérdida del brazo de aquélla supone para ésta la pérdida de su identidad. Más aún, María es siempre la misma estatua pero no siempre está compuesta de la misma arcilla: cuando pierde el brazo, la pieza de arcilla que la compone no es la misma que la componía antes.

En el caso $\beta$, a pesar de que Marta y María tienen exactamente las mismas propiedades temporales, algunos autores insisten en su diferencia. Como en el caso $\alpha$, dicen, las entidades implicadas tienen diferentes propiedades: hay enunciados abiertos modales que resultan ser verdaderos tomando a Marta como argumento, pero falsos tomando a María: ‘...podría haber sido esférica' es uno de ellos. María y Marta tienen diferentes propiedades modales. Considerarlas idénticas, pues, toparía contra nuestras más elementales intuiciones sobre la identidad, sistematizadas por la ley de Leibniz o principio de indiscernibilidad de los idénticos, cuya aplicación incluye todo tipo de propiedades. Según este famoso principio, si dos objetos, $a$ y $b$ son tales que $a=b$, entonces, para toda propiedad, $f, f(a)$ si y sólo si $f(b)$. Si $f$ es una propiedad modal, y $a$ es Marta y $b$ María, es evidente que el consecuente del condicional será falso, porque los criterios de persistencia o identidad temporal aplicados a María son mucho más restrictivos que los aplicados a Marta (hay un número menor de mundos posibles que contengan a María). Marta puede ser ovalada, parale- 
lepípeda o deforme, pero María no, puesto que perdería su identidad.

En el caso $\gamma$, el desacuerdo es racionalmente imposible, ya que, si las condiciones de persistencia de Marta son las mismas de María, Marta y María poseen las mismas propiedades modales y, dado que ocupan las mismas regiones espacio-temporales, poseen también las mismas propiedades temporales, es decir, son la misma cosa.

De entre quienes niegan que la constitución sea identidad (CNI, en adelante) podemos reconocer dos posturas. El defensor radical de CNI considera que en ningún caso la entidad constituyente es idéntica a la constituida en virtud de sus diferentes propiedades modales. Para él no hay identidad ni en el caso $\alpha$ ni en el $\beta$. La identidad de propiedades temporales en $\beta$ no es suficiente para determinar la identidad numérica de sus poseedores, que difieren en cuanto a sus propiedades modales. La identidad es una relación de todo o nada: exige indiscernibilidad en todo tiempo y en todo mundo posible.

El defensor moderado de CNI piensa que la identidad de propiedades temporales en todo tiempo es suficiente para determinar la identidad numérica de las entidades que las poseen. Aunque el caso $\alpha$ no es un caso de identidad, el caso $\beta$ sí lo es. Por supuesto que la identidad es una relación de todo o nada, pero la indiscernibilidad que exige la ley de Leibniz no incluye las propiedades modales, puesto que éstas no se predican directamente de los objetos sino relativamente al sortal o término-tipo que se les aplica. Conviene notar que el partidario de esta posición afirma la existencia de una diferencia sustancial entre el problema de la identidad modal y el de la identidad temporal, puesto que sostiene que la dependencia vigente entre la identidad de un objeto ahora y su identidad en el pasado y el futuro no vale con respecto a su identidad en el mundo real y en otros mundos posibles en que exista. 
Otros filósofos sostienen, contra todas estas razones, que la constitución es identidad (CEI). En el caso $\alpha$, es obvio que María no es idéntica tout court a Marta, en virtud de la ley de Leibniz, dado que la discernibilidad de propiedades temporales implica diferencia. Sin embargo, la diferencia de propiedades temporales no excluye la identidad en ciertas ocasiones de Marta con María: en cada momento de tiempo, María es idéntica a una cierta pieza de arcilla. Hay partes temporales de Marta idénticas a partes temporales de María. La relación de identidad es, en última instancia, una relación de todo o nada, pero ello no es incompatible con admitir una relación de identidad ocasional y contingente entre objetos persistentes. Como el partidario moderado de CNI, el defensor de CEI concibe las propiedades modales como relativas a un sortal, pero niega, contra él, que la identidad de propiedades temporales en sólo algún tiempo no baste para hablar de identidad numérica entre los objetos en cuestión.

En la literatura habitual sobre este tema, las posturas representativas vienen definidas por la del partidario radical de $\mathrm{CNI}^{3}$ frente a la del partidario de $\mathrm{CEI},{ }^{4}$ los cuales tratan los casos de identidad modal como básicamente análogos a los de identidad temporal. Aunque prima facie considero factible la postura moderada de CNI - la de quienes no los consideran análogos - no voy a examinarla en este artículo, dado que desconozco la existencia de representantes suyos que hayan elaborado una teoría ontológica que pretenda responder a todos los problemas a los que responden las anteriores y de los que trataré aquí. Los argumentos centrales que desarrollo en este capítulo contra los partidarios

3 Así lo hacen J. Perry (1970), S. Shoemaker (1970), D. Wiggins (1980) y más recientemente M. Johnston (1992).

${ }^{4}$ Cuyos más destacados representantes son A. Gibbard (1975), D. Lewis (1986) y más recientemente H.W. Noonan (1993). 
radicales de CNI, se aplican sustancialmente también a sus partidarios moderados, pero en la elaboración detallada de la posición de CNI tendré en mira autores como D. Wiggins o S. Shoemaker, partidarios de la versión radical.

Usualmente el debate entre partidarios de CNI y de CEI se presenta del siguiente modo. Quienes defienden CEI deben justificar por qué la conclusión de $\left(^{*}\right)$ no es, pese a la verdad de sus premisas, verdadera:

(*) Martha podría haber sido esférica

Martha es María

María podría haber sido esférica

El peso de la prueba recae, según parece, en quienes afirman que la constitución es identidad, ya que la ley de Leibniz exige indiscernibilidad completa para la identidad, y la diferencia de propiedades modales hace discernibles a Marta y María. ¿Cómo se justifica entonces, desde CEI, que (*) no sea un argumento válido pese a la verdad de sus premisas? La respuesta desde CEI consistirá, por supuesto, en afirmar que $\left(^{*}\right)$ es un non sequitur. Noonan (1993, pp. 134135), en esta línea, alega que los predicados modales son abelardianos, es decir, que varían de significado en función del término singular al que se aplican. Un ejemplo de tal predicado es 'así llamado en virtud de su talla', que varía de significado al aplicarse a 'Giorgione' o a 'Barbarelli'. En el caso que nos ocupa 'podría haber sido esférica' es un predicado abelardiano (en realidad, todos los predicados modales lo son): aplicada a Marta significa 'tiene una contrapartida que es una pieza de arcilla y que es esférica', pero, aplicada a María 'tiene una contrapartida que es una estatua y que es esférica'. La teoría de la contrapartida de Lewis (1971) justifica esta misma idea así: 'María' y 'Marta' 
no aparecen de modo referencialmente transparente en $\left(^{*}\right)$, de modo que no se puede aplicar lícitamente el principio de sustituibilidad de los idénticos (el correlato semántico de la ley de Leibniz). Cada término singular lleva asociado un criterio de persistencia, que fija la relación de contrapartida considerada y que relativiza la aplicación del predicado modal correspondiente. Para especificar ese criterio podemos utilizar la partícula qua. Así, Marta es el objeto qua arcilla, que podría haber sido esférica, mientras que María es el objeto qua estatua, que no podría haberlo sido. En $\alpha$ deja de existir qua arcilla antes que qua estatua. La identidad modal de un objeto es relativa al sortal (el portador del criterio de persistencia) que se considere: no hay propiedades modales de re.

Esta manera de presentar ambas posiciones no es, en vista a los objetivos que me propongo, la más satisfactoria. ${ }^{5}$ La defensa del partidario de CEI ante la falsedad de la conclusión de $(*)$ resulta ingeniosa como mucho: consigue eludir los efectos de la aplicación de la ley de Leibniz gracias a que considera los predicados modales como sui generis. Pero el de la constitución es un problema que tiene ramificaciones en lo más interno de nuestras convicciones ontológicas. Ninguna escapatoria desde la semántica puede constituir una buena respuesta a no ser que venga respaldada por la justificación de una teoría ontológica general sobre la identidad de los objetos físicos. Los partidarios de CEI pueden aportar, tanto como los de CNI, tal justificación.

Me propongo enfocar el debate de modo que tanto CNI como CEI se ofrezcan como opciones igualmente respaldadas ontológicamente en este sentido, de modo que la

5 Y que, por cierto, es muy habitual. Véanse, si no, las páginas iniciales de M. Johnston (1992) y H.W. Noonan (1993). 
presentación del problema no favorezca especialmente ni a una ni a otra.

Aparentemente el problema de la constitución parece ser independiente de una teoría ontológica general y llevarnos, en todo caso, a decidirnos por una en concreto. Sin embargo, no es así. Tanto los partidarios de CNI como los de $\mathrm{CEI}^{6}$ usan implícitamente principios que derivan de cierta concepción de la identidad ligada a cierta ontología.

Replantearé la situación problemática que nos ocupa en términos más precisos con el fin de desvelar los principios en cuestión. Diremos que 'la estatua que observamos en $\tau$ ' fija la referencia de 'María' y que 'la pieza de arcilla que compone a María en $\tau$ ' fija la de 'Marta'. Puesto que el significado de cada término se fija haciendo referencia a un momento de tiempo, parece correcto formular el problema de la constitución como el referente a si es verdad que

$$
\tau / \text { María }=\tau / \text { Marta, }
$$

donde ' $\tau / x$ ' refiere al objeto $x$ tal como ocurre en el tiempo $\tau$. Quisiera hacer algunos breves comentarios acerca de la notación. Mi propósito es que la notación no prejuzgue en absoluto la cuestión que se debate. Con ' $\tau /$ Marta' me refiero simplemente a Marta "tal y como existe en $\tau$ ". Los datos intuitivos que respaldan esta notación son los casos frecuentes en que hablamos acerca de si ese objeto en $\tau$ es el mismo que ese objeto en $\tau^{\prime}$. Tanto los partidarios de una ontología de partes temporales como sus opositores pueden aceptar esta terminología. La diferencia entre unos y otros vendrá dada porque los primeros, entendiendo ' $\tau$ /Marta' como 'Marta-en $-\tau$ ' y suponiendo que Marta exista en $\tau$ y $\tau^{\prime}$, negarán la verdad de ' $\tau$ /Marta $=\tau^{\prime}$ /Marta', mientras que los segundos la afirmarán.

6 Me refiero, por supuesto, a los partidarios prototípicos de tales posiciones: Wiggins (1980) con respecto a CNI, y Lewis (1986) con respecto a CEI. 
Concluido este paréntesis, consideremos ahora el caso $\alpha$, a saber, aquél en que Marta y María difieren no sólo modal sino también temporalmente. El partidario de CNI niega absolutamente la identidad de Marta y María, mientras que el partidario de CEI admite que, al menos en ciertos momentos de tiempo, Marta es idéntica a María. Recordemos que en este caso, debido al arrebato del escultor en, digamos, $\tau 2$, Marta deja de existir antes que María. Así, en $\tau 2$ María existía pero Marta no, por lo que:

1. $\tau 2 /$ Marta $\neq \tau 2$ /María.

Por otra parte, hay un mundo posible, digamos $M_{i}$, donde Marta es esférica, pero no María (puesto que María no es esférica en ningún mundo posible). De modo que

2. $M_{i} /$ Marta $\neq M_{i} /$ María, donde ' $M / x$ ' refiere al objeto $x$ en el mundo posible $M{ }^{7}$

De ambos hechos podemos concluir que 3 y 4 son verdaderos:

3. $\exists \tau(\tau /$ Marta $\neq \tau /$ María $)$

4. $\exists M(M /$ Marta $\neq M$ /María $)$

Según los partidarios de CNI, la verdad de 3 y 4 nos obliga a aceptar la de 5:

5. Marta $\neq$ María

Pero 3 y 4 implican 5 sólo si aceptamos 7 y 8:

7. Marta $=$ María $\rightarrow \forall \tau \tau^{\prime}\left(\tau /\right.$ Marta $=\tau^{\prime} /$ María $)$

8. Marta $=$ María $\rightarrow \forall M M^{\prime}\left(M /\right.$ Marta $=M^{\prime} /$ María $)$.

Ahora bien, los partidarios de CNI que aceptan 7 y 8 se comprometen a su vez con los principios de atemporalidad ${ }^{7}$ La interpretación de este tipo de expresión es análoga a la de ' $\tau / x$ '. 
de la identidad (AI) y necesidad de la identidad (NI), respectivamente: ${ }^{8}$

(AI) $\forall x y(x=y) \rightarrow \forall \tau \tau^{\prime}\left(\tau / x=\tau^{\prime} / y\right)^{9}$

(NI) $\forall x y\left(x=y \rightarrow \forall M M^{\prime}\left(M / x=M^{\prime} / y\right)\right)$

La pregunta es ¿es la identidad una relación necesaria y atemporal? Las intuiciones que nos dicen que en $\tau 2$ y $M_{i}$ Marta no es idéntica a María también nos dicen que, durante el tiempo en que Marta constituye la estatua y en un mundo como el descrito inicialmente, Marta es, para cada tiempo, idéntica a María, es decir, que:

$$
\begin{aligned}
& 1^{\prime} . \exists \tau(\tau / \text { Marta }=\tau / \text { María }) \\
& 2^{\prime} . \quad \exists M(M / \text { Marta }=M / \text { María })
\end{aligned}
$$

Aceptar $1^{\prime}$ y $2^{\prime}$ supone admitir los principios de ocasionalidad de la identidad (OI) y de contingencia de la identidad (CI), respectivamente:

$$
\begin{aligned}
& \text { (OI) } \exists x y \tau \tau^{\prime}\left(\tau / x=\tau / y \wedge \tau^{\prime} / x \neq \tau^{\prime} / y\right) \\
& \text { (CI) } \exists x y(x=y \wedge \exists M(M / x \neq M / y))
\end{aligned}
$$

Los defensores de CNI utilizan, pues, como premisas implícitas de su razonamiento los principios AI y NI. Quienes sostienen CEI deben aceptar, en cambio, OI y CI.

${ }^{8}$ Quiero recordar aquí de nuevo que los partidarios de CNI a que me refiero son los radicales. Un partidario moderado, al defender en este caso que Marta $\neq$ María, sólo se comprometería con (AI), no con (NI), por esencialmente las mismas razones del partidario de CEI ante la cuestión de la predicación modal. Así, desde esta postura moderada, sería posible aceptar la verdad de 'Marta $\neq$ María' y ' $\exists M(M /$ Marta $=$ $M$ /María)'.

9 La notación nos permite distinguir entre casos de identidad diacrónica (cuando $\tau \neq \tau^{\prime}$ ) y casos de identidad sincrónica (cuando $\left.\tau=\tau^{\prime}\right)$. 
Prima facie, AI y NI son lógicamente verdaderos. Me refiero al clásico argumento de Barcan-Kripke ${ }^{10}$ que reproduzco seguidamente:

1. $\forall x \square(x=x)$ (axioma)

2. $\forall x y(x=y \rightarrow \forall f(f(x) \leftrightarrow f(y))) \quad$ (ley de Leibniz)

3. $\forall x y(x=y \rightarrow(\square(x=x) \leftrightarrow \square(x=y))) \quad(f=\{y: \square(x=y)\})$

4. (NI) $\forall x y(x=y \rightarrow \square(x=y))$

(1 y lógica prop.)

$\mathrm{El}$ argumento permite igualmente deducir AI tomando $f=\{y: \forall \tau(\tau / X=\tau / y)\}$. Desde el punto de vista lógico cualquier predicado designa una clase, pero metafísicamente no resultan aceptables predicados como 'ser necesariamente (o en todo tiempo) idéntico a $x$ ', porque no denotan propiedades naturales. ${ }^{11} \mathrm{Si}$ aceptamos este tipo de argumentos para decidir cuestiones metafísicas, deberemos reconocer la validez de principios metafísicamente discutibles como el de la identidad de los indiscernibles, fácilmente demostrable a partir de 2, sustituyendo ' $f$ ' por 'ser idéntico a $x$ '. No obstante, todos $^{12}$ estaremos de acuerdo en que aceptar tal principio en función de tal propiedad lo convierte en un principio vacuo, que no recoge el contenido filosóficamente relevante del mismo.

En cualquier caso, puede ser interesante investigar el carácter modal de la identidad argumentando directamente desde el lenguaje natural, sin abordar las peculiaridades de un sistema deductivo que, en última instancia, y en especial

10 La versión de Kripke está en su "Identity and Necessity", en M. Munitz (1973, p. 136).

11 Es decir, propiedades cuyos predicados correspondientes sean candidatos a formar parte de los antecedentes o consecuentes de enunciados de leyes naturales o, menos estrictamente, propiedades tales que los objetos que las ejemplifican tienen un grado de similitud mutua suficiente.

12 O casi todos: B. Brody (1980) no lo cree así. 
al referirse a cuestiones de este calibre metafísico, pueda ser recomendable modificar. Considero que si la identidad es una relación necesaria, o bien lo es primitivamente o bien hay razones metafísicas para que lo sea. Realmente, tras la idea de la necesidad y atemporalidad de la identidad se halla una tradicional concepción ontológica acerca de la persistencia, que determina el compromiso de sus defensores con la tesis de CNI. Análogamente, que la identidad sea vista como contingente y ocasional deriva de una filosofia de la persistencia que tiene como consecuencia la tesis de CEI.

Existen dos teorías básicas sobre la persistencia. Según una de ellas, la teoría de la persistencia completa, ${ }^{13}$ los objetos persisten completos en el tiempo estando totalmente presentes en cada instante de su curso temporal. En cambio, según la teoría de la persistencia parcial, los objetos persisten sólo parcialmente en el tiempo, y tienen diferentes partes temporales en cada instante de su existencia. Diremos que los partidarios de la primera creen que los objetos ordinarios son continuantes, y los de la segunda que son procesos. Los partidarios de continuantes niegan la ocasionalidad de la identidad. ${ }^{14}$ Quienes, en cambio, entienden los objetos ordinarios como cierto tipo de

13 'Persistencia completa' y 'persistencia parcial' son los términos que usé en mi tesis doctoral para referirme respectivamente a lo que M. Johnston (1984, p. 58) denomina 'endurance' y 'perdurance'. No es lugar éste para tratar directamente el tema de la persistencia. Mencionaré simplemente que M. Johnston y S. Haslanger son partidarios de la teoría de la persistencia completa, mientras que D. Lewis y H. Noonan abogan por su rival.

14 Que si la identidad es ocasional, no es posible creer en continuantes se puede probar fácilmente. Si existen $\tau$ y $\tau^{\prime}$ tales que $\tau / O=\tau / O^{\prime}$ pero $\tau^{\prime} / O \neq \tau^{\prime} / O^{\prime}$, entonces $\tau / O \neq \tau^{\prime} / O$, puesto que $\lambda x\left(\tau / x=\tau / O^{\prime}\right) O$ pero $\lambda x\left(\tau^{\prime} / x \neq \tau^{\prime} / O^{\prime}\right) O$. Si $\tau / O$ satisface propiedades diferentes de las satisfechas por $\tau^{\prime} / O$, no pueden ser el mismo (por la ley de Leibniz). 
procesos que constan de partes temporales pueden definir una relación de identidad "más fina" restringida temporalmente, según la que, dados dos procesos unitarios $O$ y $O^{\prime}$, $\tau / O=\tau / O^{\prime}$ si y sólo si $O$ y $O^{\prime}$ se solapan en $\tau$ (tienen una parte temporal común en $\tau$ ). Análogamente para el caso modal. La metafísica ligada a las tesis OI y CI nos permite, pues, aceptar que:

$\tau 2 /$ Marta $\neq \tau 2$ /María

aunque, antes, cuando en $\tau$ l María estaba intacta:

$\tau$ l/Marta $=\tau$ l/María.

Para un partidario de CEI, en cada momento de tiempo en que María existe, existe una pieza de arcilla, que la constituye y a la que es idéntica. Así pues, (\#) es verdadero:

(\#) $\forall \tau(\tau /$ María existe $\rightarrow$ (existe una $\tau /$ pieza: Cons $(\tau /$ pieza, $\tau /$ María $) \wedge \tau /$ pieza $=\tau /$ María $))$

Un partidario de CNI negaría, por supuesto, (\#), en virtud de las diferentes propiedades temporales de María y las sucesivas piezas de arcilla que la componen (pensemos simplemente en que la pieza de arcilla que compone a María en $\tau 2$ era una parte espacial propia de Marta en $\tau 1$, pero, por supuesto, María en $\tau$ l no era una parte espacial propia de Marta).

Quienes aceptan CNI en su versión radical se comprometen con la necesidad y atemporalidad de la identidad y también con una teoría de continuantes, mientras que los defensores de CEI, al aceptar la contingencia y ocasionalidad de la identidad, deben aceptar una teoría de procesos.

II

Veamos ahora cuál es el abanico de concesiones que supone aceptar CNI. Si María no es idéntica a Marta, ¿qué relación 
se da entre ellas? La relación de constitución. Marta, la arcilla, constituye o compone a María, la estatua, ${ }^{15}$ pero no es idéntica a ella. Es cierto que Marta es María, pero en el sentido constitutivo del verbo 'es', no en el de identidad numérica. La relación de constitución es claramente una relación relativa a un tiempo en el sentido geacheano de la expresión: ' $x$ constituye a $y$ en $t$ ' no puede analizarse como ' $x$ constituye a $y \wedge x$ e $y$ existen en $t$ '. La materia que constituye a un objeto varía según el momento de tiempo. Si el 'es' del enunciado del que tratamos es un 'es' de constitución, todo defensor de la atemporalidad de la identidad tendrá una buena razón para negar que sea, también, un 'es' de identidad.

Se han dado dos interpretaciones básicas del 'es' de constitución. Según la primera, (A), 'Marta es María' ha de entenderse como 'Marta constituye a María'. Johnston (1992) adopta la distinción lockeana, ${ }^{16}$ según la cual Marta y María son entidades de categoría lógica diferente. Marta es una simple masa de materia, mientras que María es un objeto articulado, de modo que, aunque Marta constituye a María, María no podría constituir a Marta. La relación de constitución es irreflexiva y asimétrica. La diferencia de categoría entre masas y objetos se puede justificar así: un objeto es una unidad estructurada; las unidades estructuradas arquetípicas son las unidades naturales; los objetos que son unidades naturales caen bajo ciertos sortals de sustancia conocidos como 'géneros naturales', los cuales "determinan, para $x$, para $y$, y para todo otro miembro del género, un principio de actividad". ${ }^{17}$ Lo que distingue un objeto

15 Ya decía Aristóteles (Metafísica VII, 1033a 5-9) que una estatua no es piedra sino de piedra.

16 En su Essay Concerning Human Understanding, libro II, cap. XXVII. Véase Locke (1993, pp. 174-177).

17 D. Wiggins (1980, p. 77). 
de una mera masa es, pues, no su constitución material, sino que en el primero esté definido un principio unitario de funcionamiento, actividad u organización. El contenido del término 'constituir', en su uso cotidiano, presenta las propiedades formales que Johnston atribuye al concepto de constitución al que apela, y la justificación metafísica de dicho uso semántico habitual bien podría deberse a la diferencia categorial a que Johnston, como ya Locke, se refiere.

Otra posibilidad, (B), consiste en interpretar 'Marta es María' como 'Marta está constituida por la misma materia que María'. Así parecen entenderlo, mutatis mutandis, Shoemaker (1970, pp. 530-531), D. Robinson (1982a, 320, n. 4)(1982b) y Wiggins $(1980,197$, n. 1.19). La relación 'estar constituido por la misma materia que' es reflexiva y simétrica. ${ }^{18}$ Según esta interpretación, Marta y María pertenecen ambas a la categoría de objetos, sus sortals respectivos son diferentes (no podrían ser iguales, ya que, por ejemplo, si ambas fueran estatuas, tendrían las mismas propiedades modales, con lo cual sería incontrovertible su

18 Tengo mis reservas acerca de la pertenencia o no de Wiggins a este grupo. Sus declaraciones sobre el tema de la constitución son tan interesantes como oscuras. Por un lado, Wiggins parece creer que la relación de constitución es simétrica cuando afirma que su uso de 'constituye' es un uso mereológico del término y que "la identidad es un caso especial de constitución". Sin embargo, en otros textos (véase la nota 24 de este capítulo), declara que su intención al distinguir 'constitución' de 'identidad' es la de distinguir diferentes categorías de objetos, en el sentido marcado por la posibilidad (A), lo cual es claramente incompatible con la simetría de la relación, aunque no con su no asimetría, entendiendo la relación técnica de 'constitución' como una ampliación de la cotidiana. Mis razones para incluirlo entre los partidarios de la posibilidad (B) se basan en dos datos: el primero, un dato de autoridad, que se debe a una declaración de Robinson (1982a, 320 , n. 4) al respecto. El segundo, un dato de prioridad, que consiste simplemente en que es en su Sameness and Substance donde Wiggins trata con más detalle el tema de la constitución y en este libro se sugiere más claramente la interpretación que le atribuyo. 
identidad) y están constituidas por una tercera entidad. El término 'constitución' adquiere aquí un claro sentido técnico que lo distancia de nuestro cotidiano 'estar hecho de'.

Ambas posibilidades suponen admitir la coincidencia de varias entidades. Diremos que $x$ coincide con $y$ en $t$ si y sólo si:

(i) $x \neq y$

(ii) $x$ ocupa en $t$ totalmente la misma región espacial que $y$.

(A) sólo nos obliga a aceptar la coincidencia de dos entidades de diferente categoría; (B) tiene la desventaja de admitir la coincidencia de, por lo menos, tres entidades, dos de ellas de la misma categoría. Las tres entidades involucradas en el problema serían dos objetos y una masa: la estatua (María), la pieza de arcilla (Marta) y la arcilla. La arcilla tiene condiciones de persistencia menos restrictivas que la pieza de arcilla. Si le corto un brazo a Marta y lo dejo sobre la base, no tengo la misma pieza de arcilla, pero sí la misma arcilla. La pieza de arcilla es un agregado de silicatos, pero la arcilla no es más que una mera colección de los mismos, que sigue existiendo a pesar de que se dispersen. ${ }^{19}$ No le es esencial sino cierta estructura molecular de sus partes, sea cual sea la organización de las mismas. Al agregado le es esencial, además, que sus partes tengan cierta cohesión interna. La estatua, a diferencia del agregado y de la colección, es un objeto articulado: posee no sólo esa estructura molecular y esa cohesión entre sus partes, sino también cierta articulación esencial de la que ambas carecen (en este caso, la necesaria para determinar

19 Sobre el tema de la categoría de las masas de materia, colecciones y montones de objetos, véase H. Laycock (1972) y Hirsch (1976), quienes defienden un enfoque meramente mereológico de la materia (materia como una mera colección de elementos). El lugar clásico es, por supuesto, Aristóteles (Metafísica X). 
cierto contorno). Por un lado, los agregados y los objetos articulados poseen cierta cohesión interna (en virtud de las relaciones causales que mantienen sus partes espaciales) ${ }^{20}$ de que carecen las colecciones. Este rasgo hace a ambos merecedores del nombre de 'objetos'. Por otro lado, para los agregados y las colecciones vale el principio del esencialismo mereológico: pierden su identidad al perder una de sus partes componentes. En cambio, con respecto a los objetos articulados, no es razonable, de entrada, creer que les sea esencial la posesión de todas sus partes. ${ }^{21}$

Esta categorización de entidades en términos de objetos articulados/ agregados/ colecciones me permite justificar las diferencias de que intuitivamente hablamos cuando nos referimos a estatuas/ piezas de arcilla/ arcilla. Cada tipo de entidad tiene condiciones de persistencia diferentes. Podemos comprobarlo pensando en diferentes situaciones contrafácticas con el ejemplo de Marta y María.

Consideremos un mundo posible, $N$, donde la arcilla que se usó en el mundo real $M$ para modelar una estatua femenina sea modelada como retrato ecuestre de Fidel Castro. En ese mundo existe la misma arcilla y la misma pieza de arcilla, pero la estatua es diferente. Un mundo posible, $Q$, en que secciono el brazo izquierdo de la estatua, es un mundo posible donde la estatua y la arcilla son las mismas, pero donde la pieza de arcilla no. En $L$, donde fundo en bronce la estatua y descompongo químicamente la arcilla, la estatua es la misma (quien no lo crea, tendrá que negar que $E l$ beso, tal y como se le conoce, sea efectivamente obra de Rodin), pero no existen ni la arcilla ni la pieza de arcilla.

20 La idea de tal unidad causal puede hallarse en M. Slote (1979).

21 R. Chisholm (1973) no lo consideraría razonable. Según él, los entia per se, aquellos con respecto a los que cabe hablar de identidad estricta, poseen esencialmente todas sus partes componentes. 
$M, N, Q$ y $L$ son mundos posibles diferentes que ilustran las diferentes condiciones de persistencia de objetos articulados, agregados y colecciones. De estos tres tipos de entidades, una colección de tipo $f$ es la entidad con condiciones de persistencia más laxas, cuyas propiedades esenciales vienen definidas mereológicamente y por su pertenencia a $f$. Un agregado de tipo $f$ es una entidad de condiciones de persistencia intermedias, cuyas propiedades esenciales vienen definidas mereológicamente y por cierta cohesión interna causal entre sus partes componentes de tipo $f$. Un objeto articulado de tipo $f$ es la entidad con condiciones de persistencia más estrictas, cuyas propiedades esenciales no vienen definidas mereológicamente y que incluyen no sólo cierta cohesión interna causal entre sus partes componentes, sino también cierta articulación de las mismas fijada por su pertenencia al tipo $f$. En función de la cohesión interna causal entre las partes componentes que se da tanto en un agregado como en un objeto articulado, parece lícito calificarlas a ambas de 'objetos'.

Pues bien, una vez especificadas las condiciones de persistencia de los tres tipos de entidades, conviene hacer marcha atrás para recuperar la cuestión que se estaba considerando. ¿Aceptar la lectura propuesta por (B) nos obliga a creer en la coincidencia o coexistencia en el mismo espaciotiempo de las tres entidades? Desde luego que sí, sólo que, en realidad, no sólo la de tres, sino, como se puede mostrar fácilmente, la de cuatro entidades. Si la diferencia de condiciones de persistencia es suficiente para justificar la distinción entre objetos, entonces, a la distinción objeto articulado/ agregado/ colección hemos de añadir un cuarto elemento: la materia. Las colecciones de elementos a las que denominamos con términos de masa como 'arcilla', 'hierro' o 'agua' corresponden a la categoría de lo que llamamos 'material'. Las condiciones de persistencia de un material (la 'materia segunda' aristotélica, en algún sentido 
de 'aristotélica') son más restrictivas que las de la mera materia (la 'materia prima' aristotélica), puesto que ésta permanece en cualesquiera combinaciones estructurales en que se halle dispuesta. ${ }^{22}$ Hablando con propiedad, pues, Marta, la pieza de arcilla, no es una pieza de materia, sino una pieza de material.

(B), por tanto, supone aparentemente una sobrecarga ontológica mayor que (A), con la que pocos quieren comprometerse. Sin embargo, si las distinciones presentadas son válidas, también los partidarios de (A) deberían comprometerse con la coincidencia de cuatro entidades, sólo que no todas ellas serían de la misma categoría. La materia y el material serían calificados de masas coincidentes con los objetos que serían la pieza de material y la estatua.

Los filósofos partidarios de CNI han puesto restricciones a esta múltiple coincidencia. Wiggins (1968) formula un principio según el cual no pueden coincidir dos entidades que caigan bajo el mismo sortal, principio que pretende estar de acuerdo con el de Locke, según el cual dos objetos del mismo tipo no pueden ocupar el mismo espacio al mismo tiempo. ${ }^{23}$ Johnston (1993) cree que el principio debe impedir la coincidencia de entidades de la misma categoría. ${ }^{24}$

22 Para una clarificación muy satisfactoria de la distinción entre pieza de material/ material/ materia, véase C. Hughes (1994), quien se sirve de ella con fines distintos de los míos.

23 Lowe (1983) y Simons $(1987,6.3$.) siguen a Wiggins. Hay autores que incluso llegan a afirmar que puede darse coincidencia entre objetos del mismo sortal. Shorter (1977) escoge, para mostrar que dos objetos del mismo tipo pueden coincidir, el ejemplo de dos carreteras que se juntan. En realidad, es fácil descalificar el ejemplo diciendo que sólo hay un objeto físico que, por razones convencionales o históricas, se ha dado en denominar de modo diferente por lo que respecta a ciertos tramos o partes suyas. Simons (1985) presenta su ejemplo de los $n$ montones. D. Robinson (1982a) cree que los casos de fisión justifican la coincidencia prefisiónica de dos o más objetos que caen bajo el mismo sortal.

24 Ésta es también la idea de Wiggins, si nos atenemos a lo que 
Sin embargo, ¿qué justificación ofrece Johnston para ello? Si aceptamos, como se da por supuesto desde CNI, que la diferencia de propiedades modales es condición suficiente para la diferencia de entidades, ¿qué razón hay para negar que la estatua y la pieza de material coinciden? Son de la misma categoría, son objetos, aunque uno sea un objeto "socialmente relevante" y el otro un mero agregado. La pieza de arcilla no es una mera masa. A diferencia de la arcilla, es una colección de silicatos con cierta cohesión o unidad interna. Quizá se diga que una pieza de arcilla tiene condiciones de persistencia demasiado laxas como para ser considerada un objeto. Personalmente creo que el que lo sea o no depende más de nuestros intereses clasificatorios que de cómo sea el mundo: un chicle no deja de ser un objeto porque sea más maleable y persista en más diversas formas que una goma de borrar. No obstante, incluso aunque negáramos la categoría de objeto a la pieza de arcilla, el partidario de (A) debería admitir igualmente la coincidencia de cuatro entidades: un objeto y tres masas. Toda una población, fruto de una visión multifacetada de la realidad.

Así pues, tanto si aceptamos (A) como (B), CNI lleva a la tesis de la coincidencia, ${ }^{25}$ según la cual toda región espacio-temporal está completamente ocupada por dos o más entidades cualitativamente idénticas. El ejemplo siguiente nos ayudará a ver con más nitidez la peculiaridad de esta tesis.

dice, refiriéndose a su ejemplo del árbol y el agregado de moléculas de celulosa que lo compone, en Wiggins $(1968,92)$ : "lo que he querido mostrar con este simple ejemplo no es totalmente diferente de una tesis que se ha convertido en lugar común en filosofía, y según la cual se defiende que cosas como árboles y los agregados que 'son' (o en los que consisten) pertenecen a tipos lógicos diferentes".

25 Chappell (1990) la llama 'tesis de la doble existencia', prejuzgando el número de entidades con que se compromete el partidario de CNI. Por lo que hemos visto sería más apropiado llamarla 'tesis de la cuádruple existencia', por lo menos. 
Si hemos llamado 'María' a la estatua, llamemos 'Mar' a la parte de la estatua que es exactamente ella misma excepto su brazo izquierdo. ${ }^{26}$ Mar es ahora, en $t l$, una parte propia de María. Pero ¿qué ocurriría si en $t 2$ alguien le quiebra a María su brazo izquierdo (caso $\alpha$ )? (donde $t 2<t 3$ ). Si decimos que en $t 3$ (donde $t 2<t 3$ ) Mar no es idéntica a María, entonces hemos de admitir, tras la sustracción, la coincidencia de dos entidades. ¿Cómo es que, cuando María pierde el brazo, dos entidades diferentes ocupan el mismo lugar?

El interés del caso Mar-María frente al de Marta-María radica en que en el segundo los términos singulares implicados podían referir a entidades de diferente categoría, mientras que en el primero los términos singulares implicados parecen poder referir ambos a entidades de la misma categoría. En $t$ l Mar no es la arcilla de la que está hecha una parte de María sino la pieza de arcilla o la estatua que es esa parte. Hay objetos de tipo $f$ algunas de cuyas partes espaciales son $f$ : una corona es una joya y está compuesta por joyas. ¿Por qué no admitir que algunas de las partes de una estatua son estatuas? Mar y María serían estatuas diferentes pero no distintas: serían temporalmente idénticas al solaparse en $t 3$. Al fin y al cabo, dado que Mar ocupa el mismo espacio que María y es cualitativamente idéntica a ella tras la sustracción, ¿por qué negar su identidad numérica?

El partidario de CNI aduce la diferencia de propiedades temporales: María ha perdido en $t 3$ una propiedad que antes tenía, a saber, la de "tener un brazo izquierdo", mientras que Mar no ha sufrido cambio alguno. Así pues, Mar

26 La estructura del ejemplo que presento está basada en un caso inspirado en el original de Guillermo de Sherwood. 
y María, si fueran idénticas, serían discernibles, ${ }^{27}$ lo cual es absurdo. Por supuesto, el partidario de CNI suele negar que Mar sea un objeto: en $t 1$ Mar constituye a una parte de María, y en $t 3$ la constituye por completo. La relación de constitución no es de identidad: Mar es simplemente la masa que constituye al objeto María. Pero ¿qué justifica esa diferencia de categoría? En $t 3$, Mar tiene, al fin y al cabo, el mismo principio de actividad que María. Sin embargo, reconozco que resulta problemático en un sentido no relevante al caso, hablar de principios de actividad de objetos artificiales como estatuas. Para evitar este escollo inútil en la argumentación, expondré un caso análogo que haga referencia a un objeto natural.

Supongamos que 'Tilo' refiere a una planta con hojas y 'Til' a su tallo. Si en $t 3$ Tilo ha sido reducido a Til, ¿no diremos que Tilo es en $t 3$ idéntico a Til? ¿Acaso no tienen el mismo principio de actividad? Para el partidario radical de CNI, incluso aunque Tilo y Til hubieran sido creados y destruidos sintéticamente y compartieran todas sus propiedades temporales, a pesar de ello, seguirían siendo diferentes, en virtud de sus diferentes propiedades modales: Til no podría tener hojas, pero Tilo sí. Para que $x$ y $y$ sean el mismo se exige que ocupen las mismas regiones espacio-temporales en todo mundo posible.

El problema de la coincidencia no se debe sólo a esta extraña sobrepoblación, sino a la dificultad para restringirla. Johnston nos decía que nunca pueden coincidir dos

27 Una excelente exposición de las tesis que se ponen en juego al tratar este problema se encuentra en Simons (1987, pp. 119 y ss.). Para los defensores de CNI, el ejemplo pone en duda la transitividad de la identidad. Supongamos que en $\tau 2$ se hace la amputación. Si $\tau 1 /$ María $=$ $\tau 3 /$ María, $\tau 1$ /Mar $=\tau 3$ /Mar, y $\tau 3 /$ Mar $=\tau 3$ /María, entonces $\tau 1 /$ Mar $=$ $\tau$ l/María, lo cual es falso. También se puede entender como poniendo en duda el principio de no-contradicción o la ley de Leibniz, como indico en el texto. Todos ellos son diferentes caminos para llegar a la misma conclusión. 
entidades del mismo sortal ni de la misma categoría. Pero hemos visto que tanto la pieza de arcilla como la estatua pueden ser considerados objetos, y Tilo y Til, una planta y su tallo respectivamente, son objetos también. Si, como dice Wiggins $(1968,92)$, decir que la constitución no es identidad supone señalar los diferentes "tipos lógicos" a los que unas y otras entidades pertenecen, reconocer la obligación del partidario radical de CNI a negar tal diferencia en los casos relevantes pone serias dudas en la corrección de su teoría.

Por otro lado, recordemos que la única justificación que desde la postura radical de CNI se ofrece para aceptar la sobrepoblación de entidades coincidentes está en la diferencia de propiedades modales. En mi opinión, tal diferencia no es suficiente para justificar la coincidencia. La razón es que atenta contra un razonable principio de superveniencia, según el cual las propiedades modales supervienen sobre las no modales.

Pensemos en una propiedad disposicional como la solubilidad. El azúcar tiene esa propiedad y el mercurio no. El azúcar posee la propiedad de la solubilidad debido a que posee una peculiar estructura microfísica de la que carece el mercurio. Esa estructura microfísica puede ser o bien común a todo objeto soluble, o bien miembro de la familia de estructuras que realizan la solubilidad. En cualquier caso, el mercurio no posee ninguna de ellas. Lo importante es que, gracias a esa estructura, el azúcar es soluble, y que, debido a la ausencia de esa u otras estructuras de la familia, el mercurio no lo es.

En general, si un objeto difiere de otro porque posee diferentes propiedades modales que él, esperamos racionalmente que difiera también en propiedades de otro nivel más básico, por así decirlo. Las piedras no pueden hablar, decimos, porque, entre otras cosas, carecen de órganos fonadores. Explicamos nuestra atribución de propiedades 
modales a los objetos fundamentándola en atribuciones de propiedades de otro nivel.

Considero conveniente, por tanto, formular y aceptar el siguiente principio de superveniencia de las propiedades modales con respecto a las no modales:

La familia de propiedades modales $M$ superviene sobre la familia de propiedades no modales $N$ si y sólo si necesariamente para toda propiedad $f$ de $M$, si un objeto $x$ tiene $f$, entonces existe una propiedad $g$ de $N$ tal que $x$ tiene $g, y$ cualquier $y$ que tenga $g$ tiene $f$.

Se trata de un principio de superveniencia fuerte, es decir, tiene validez nómica, no meramente fáctica. ${ }^{28} \mathrm{La}$ carencia de órganos fonadores justifica la no posesión de ciertas propiedades modales por parte de las piedras en este y cualesquiera otros mundos metafísicamente posibles.

El principio de superveniencia en cuestión no es reductivo. Aunque lo más razonable, a mi entender, sería que lo fuera, creerlo así no afecta a la cuestión que tratamos. Por otro lado, ha de quedar claro que el sentido en que he usado la denominación 'no modal' no es un sentido estrictamente técnico sino más bien intuitivo. Propiedades no modales son ser blanco o ser simpático. Este sentido es compatible con la idea de que, en realidad, todas las propiedades son, en cierto grado, modales, puesto que se pueden ver como poderes causales condicionales: ser blanco es ser capaz de reflejar todos los colores del espectro al ser iluminado de tal y tal modo. ${ }^{29}$ En ese caso, el principio de superveniencia citado podría reformularse de modo que exigiera la superveniencia de las propiedades que son poderes causales de

28 Con respecto a la diferencia entre superveniencia fuerte y débil, véase J. Kim (1984).

29 Shoemaker explicita la concepción de una propiedad como cierto tipo de disposición en su "Causality and Properties", en Inwagen (ed.), 1980. 
primer orden con respecto a las propiedades que son poderes causales de segundo orden. Así, estar afilado es un poder causal de segundo orden en la medida en que es un poder para producir otro poder de primer orden, a saber, el poder de cortar la mantequilla dadas ciertas condiciones. ${ }^{30}$

Si CNI es una tesis válida, el principio de superveniencia citado tiene excepciones gratuitas en todos los casos de coincidencia. Si María hubiera sido modelada al unir dos piezas de arcilla separadas que juntas hubieran formado a Marta, Marta y María seguirían teniendo diferentes propiedades modales, y seguirían siendo diferentes aunque fueran simultáneamente destruidas (el caso $\beta$ ). Sin embargo, tendrían exactamente las mismas propiedades no modales durante toda su existencia. ¿En base a qué se tendrían, pues, diferentes propiedades modales? Incluso aunque Marta fuera formada antes que María (supongamos que en $t 1$ existe Marta, pero aún no María), sus diferentes propiedades no modales (de Marta: "nacer en $t 1$ ”, "ser grisácea en $t 1$ "; de María: "no nacer en $t 1$ ", "no ser grisácea en $t 1$ ") no constituyen una base de superveniencia apropiada para sus diferentes propiedades modales (de Marta: "poder ser esférica", "no poder perder una parte componente"; de María: "no poder ser esférica", "poder perder una parte componente").

Si no es la diferencia de propiedades no modales lo que justifica la diferencia entre Mar y María tras la sustracción, ¿qué es lo que la justifica? Se ha razonado que Mar y María no sean idénticas en todo momento de tiempo, pero ¿qué las hace diferentes en cada momento de tiempo a partir de $t 2$ ? No se trata tan sólo de que si aceptamos CNI se produzca una duplicación innecesaria de entidades, sino de

30 Véase al respecto el apartado IV del artículo citado en la nota anterior. 
que no hay una base seria para formular, de modo directo, un criterio de diferencia, digamos 'simultánea', entre cada par. Wiggins se ve forzado a decir que Marta y María son, para cada momento de tiempo en que coinciden, cualitativamente idénticas y, a pesar de que ocupan simultáneamente el mismo espacio, son diferentes. Wiggins no parece poder aportar el criterio de su diferencia, apoyándose en sus ideas sobre la identidad. Para él, la identidad de un objeto en un tiempo depende de hechos anteriores y posteriores a ese tiempo: la diferencia de hechos extrínsecos al tiempo en consideración es suficiente para justificar la diferencia en el mismo. Wiggins piensa, quizá, que este principio se sustenta en la idea de que la identidad es una relación de "todo o nada": identidad es identidad en todo tiempo y en todo mundo posible. Para los partidarios de CEI, la relación de identidad como relación de "todo o nada" es compatible, sin embargo, con la idea de que la identidad de un objeto en un tiempo es independiente de hechos extrínsecos al mismo, gracias a la segmentación más fina de la realidad que ofrece la teoría de partes temporales.

\section{III}

En el apartado anterior mostré que aceptar CNI exige aceptar el principio de coincidencia y violar el principio de superveniencia de las propiedades modales respecto de las no modales. En este apartado presentaré la alternativa a CNI, a saber, CEI, y su modo de eludir las críticas que pueden suscitar los compromisos adquiridos por su rival.

Desde CEI es lícito sostener que María, la estatua, es idéntica a Marta, la pieza de arcilla, en ciertos tiempos y en ciertos mundos posibles, puesto que en ellos son cualitativamente idénticas y ocupan los mismos espacios en los mismos tiempos. El objeto señalado qua estatua tiene las mismas propiedades modales que María, pero diferentes 
de las del objeto qua pieza de arcilla. El objeto sin "etiquetar", es decir, como mero referente del acto de ostensión, no está esencialmente articulado de cierto modo, pero sí el objeto qua estatua. No es, por tanto, contradictorio que un mismo objeto tenga diferentes criterios de persistencia, porque ningún criterio de persistencia es atribuible al objeto "desnudo" sin más. Puesto que la diferencia de criterios de persistencia no justifica la coincidencia, la estatua, la pieza de material, el material y la materia no son diferentes entidades sino simplemente la misma.

Marta y María son dos objetos tetradimensionales diferentes, sumas de partes temporales que se solapan parcialmente. Cuando María ha perdido el brazo izquierdo, Marta no es idéntica a María. Pero antes de la pérdida, Marta es idéntica a María: es ocasional y contingentemente idéntica a ella (vid. apartado I). Lo es derivativamente, en cuanto que una parte temporal de Marta es estrictamente idéntica a una parte temporal de María. Hasta la pérdida del brazo, Marta ocupa el mismo espacio en el mismo tiempo que María y es cualitativa y numéricamente idéntica a ella. Como objetos tetradimensionales o procesos, Marta y María no son idénticos en todo momento, pero es posible definir una relación de identidad restringida a un tiempo, según la cual tiene sentido decir que son ocasionalmente idénticos. Tampoco son idénticos en todo mundo posible, pero, si María hubiera surgido de reunir dos trozos de arcilla que se destruyeran al tiempo que Marta (caso $\beta$ ), entonces Marta y María serían contingentemente idénticas.

Dado un objeto y la pieza de materia que lo constituye, recordemos los dos tipos relevantes de ejemplos que pueden presentarse:

a. Que el objeto comparta sólo en ciertos momentos de tiempo todas sus propiedades temporales con la pieza. 
b. Que el objeto comparta siempre todas sus propiedades temporales con la pieza.

Pues bien, en b el objeto y la pieza son idénticos en el mundo real. Comparten todas sus propiedades excepto las modales, que los hacen diferentes, no en este mundo donde su curso temporal es el mismo, sino en otros mundos posibles donde sus contrapartidas son diferentes: la arcilla que en el mundo real, $M$, es idéntica a la estatua, es idéntica en otro mundo posible, $M^{\prime}$, a un jarrón, y análogamente, la estatua que en $M$ es de arcilla en $M^{\prime}$ es de mármol.

En a sólo un fragmento espacio-temporal del objeto es idéntico a la pieza que lo constituye. Ciertas partes temporales de la pieza no son respectivamente idénticas a los objetos que constituyen. La diferencia de propiedades modales se refleja en las diferentes contrapartidas, análogamente a como la diferencia de propiedades temporales es la explicación de las diferentes partes temporales. La identidad de un objeto en un momento de tiempo no ha de venir definida por sus propiedades modales y temporales pasadas o futuras, sino por las propiedades que posee en ese mismo momento de tiempo. Entendiendo propiedad como poder causal, está claro que la estatua y la pieza de arcilla tienen, en ciertos tiempos, los mismos poderes causales, y eso basta para afirmar su identidad en ellos.

Con respecto al caso Mar-María, la solución desde CEI sería la siguiente. Citemos una versión del argumento que usualmente se presenta para convencernos de CNI:

i. $\quad \tau$ l/María $=\tau 3$ /María $\quad$ (persistencia de María)

ii. $\tau 1 / \mathrm{Mar}=\tau 3 / \mathrm{Mar} \quad$ (persistencia de Mar)

iii. $\tau 3 /$ Mar $=\tau 3 /$ María $\quad$ (supuesto de CEI)

iv. $\tau$ l/Mar $=\tau$ l/María

(transitividad de la identidad en 1, 2 y 3 ) 
Es decir, si tras la sustracción en $\tau 2$, Mar es María en $\tau 3$ (según CEI), dado que tanto María como Mar persisten (i y ii), por transitividad de la identidad, Mar y María deberían ser idénticas también en $\tau$ l, antes de la sustracción. Pero eso no es cierto, de modo que uno de los supuestos es incorrecto, y se nos recomienda sospechar de iii, de modo que CNI.

Sin embargo, el partidario de CEI no tiene que negar iii ni tampoco poner en duda la transitividad de la identidad para negar la validez del argumento. Simplemente, desde su ontología procesualista, niega la verdad de las premisas i y ii. Mar y María no son continuantes, ambas persisten de $\tau 1$ a $\tau 2$, pero su persistencia es parcial (no, como se presupone en i e ii, completa) de modo que ' $\tau$ l/Mar' refiere a una parte temporal diferente de ' $\tau 2 /$ Mar', e igualmente en el caso de ' $\tau$ l/María' y ' $\tau 2 /$ María'.

La solución de CEI al problema de la constitución es, en mi opinión, superior en simplicidad a la ofrecida por CNI. ${ }^{31}$ El problema de la diferencia, derivado del de la coincidencia, no surge desde esta óptica. El caso Mar-María no presenta reto alguno al principio de superveniencia de las propiedades modales respecto de las no modales. Las propiedades modales no son relevantes a la hora de juzgar la identidad de Mar y María en el mundo real. Nuestras intuiciones sobre casos de identidades ocasionales y contingentes quedan bien reflejadas en la teoría de la contrapartida, una teoría compatible con las tesis en que CEI se basa. Aceptar CEI tiene desde luego un precio: entender los objetos como procesos con partes temporales, cuyas propiedades modales no se predican directamente de ellos,

31 “Quizá sí en simplicidad — puede alegar un partidario de CNIpero no en economía, puesto que, desde CEI, cada objeto consta de muchísimas partes temporales." 
que carecen per se de propiedades esenciales. En cualquier caso, qué precio sea realmente más costoso depende de consideraciones metafísicas de más vasto alcance del que este artículo se puede permitir.

\section{BIBLIOGRAFÍA}

Aristóteles, Metafísica, en García Yebra, V. (comp.), Gredos, Madrid, 1982.

Brody, B., 1980, Identity and Essence, Princeton University Press, Princeton.

Burke, M.B., 1992, "Copper Statues and Pieces of Copper: a Challenge to the Standard Account", Analysis, 52, pp. 1317.

Chappell, V., 1990, "Locke on the Ontology of Matter, Living Things and Persons", Philosophical Studies, 60, pp. 19-32.

Chisholm, R.M., 1973, "Parts as Essential to their Wholes", The Review of Metaphysics, 26, pp. 581-603.

French, P.A., T.E. Uehling y H.K. Wettstein (comp.), 1979, Midwest Studies in Philosophy, University of Minnesota Press, Minneapolis, vol. 4.

Gibbard, A., 1975, "Contingent Identity", Journal of Philosophical Logic, 4, pp. 187-221.

Heller, M., 1990, The Ontology of Physical Objects. Four Dimensional Hunks of Matter, Cambridge University Press, Cambridge.

Hirsch, E., 1976, "Physical Identity", The Philosophical Review, LXXXV, 3, pp. 357-389.

Hughes, C., 1986, "Is a Thing Just the Sum of its Parts?", en Proceedings of the Aristotelian Society, pp. 213-233.

— 1994, "The Essentiality of Origins and the Individuation of Events", The Philosophical Quarterly, 44, 174, pp. 26-44.

Inwagen, P.V. (comp.), 1980, Time and Cause, Reidel Publishing Company.

Johnston, M., 1984, Particulars and Persistence, tesis doctoral, University Microfilms International, Princeton University. 
Kim, J., 1984, "Concepts of Supervenience", Philosophy and Phenomenological Research, XLV, 2, pp. 153-176.

Kripke, S., 1980, "Identity and Necessity", en Inwagen (comp.), pp. 135-164.

Laycock, H., 1972, "Some Questions of Ontology", The Philosophical Review, 81, pp. 3-42.

Lewis, D., 1971, "Counterparts of Persons and Their Bodies", The Journal of Philosophy, 68, pp. 203-211.

, 1986, On the Plurality of Worlds, Basil Blackwell, Oxford.

Locke, J., An Essay Concerning Human Understanding, Everyman, Londres, 1993.

Lowe, E.J., 1983: "Instantiation, Identity and Constitution", Philosophical Studies, 44, pp. 45-59.

Munitz, M.K., et al. (comp.), 1973, Logic and Ontology, New York University Press, Nueva York.

Noonan, H.W., 1993, "Constitution is Identity", Mind, 102, pp. 133-145.

Perry, J., 1970, “The Same F", The Philosophical Review, 80, pp. $181-200$.

Robinson, D., 1982a, "Reidentifying Matter", The Philosophical Review, 91, pp. 317-341.

- 1982, The Metaphysics of Material Constitution, tesis doctoral, University Microfilms International, Monash University.

Shoemaker, S., 1970, "Wiggins on Identity", The Philosophical Review, 79, pp. 529-544.

—, 1980, "Causality and Properties", en Iwagen (comp.), pp. 109-135.

Shorter, J.M., 1977, "On Coinciding in Space and Time", Philosophy, 52, pp. 399-408.

Simons, P., 1985, "Coincidence of Things of a Kind", Mind, 94, pp. 70-75.

- 1987, Parts. A Study in Ontology, Clarendon Press, Oxford, partes 1 y 2 .

Slote, M., 1979, "Causality and the Concept of a "Thing"", en French, P.A., et al., pp. 387-400. 
Wiggins, D., 1968, "On Being in the Same Place at the Same Time", The Philosophical Review, 77, pp. 90-95.

—_ 1980, Sameness and Substance, Basil Blackwell, Oxford.

Recibido: 29 de enero de 1996 


\section{SUMMARY}

This paper is concerned with the notions of constitution and identity. It has a threefold purpose. First, to examine and evaluate the two main positions (CII, CNI) that in recent discussion are supposed to be the answers to the puzzles raised on this topic. I will try to show its commitments with certain views on the modal and temporal character of the identity relation. Second, to show that the classical position (CNI) suggest some criticisms because of its acceptance of an antioccamist coincidence principle that contravenes a reasonable principle of supervenience. Finally, I want to stress the capacity of the rival theory of dealing with those problems. 